\title{
Communication
}

\section{Fabrication of Piezo-Resistance Composites Containing Thermoplastic Polyurethane/Hybrid Filler Using 3D Printing}

\author{
Kyoungho Song $\mathbb{D}^{\mathbb{B}}$, Hansol Son, Suwon Park, Jonghan Lee, Jungsik Jang $\mathbb{D}$, Mijung Lee and Hyun-joo Choi * \\ Department of Materials Science and Engineering, Kookmin University, Seoul 02707, Korea; \\ 4byeol@kookmin.ac.kr (K.S.); tjseodkr@kookmin.ac.kr (H.S.); tndnjs211@kookmin.ac.kr (S.P.); \\ ljh06_19@kookmin.ac.kr (J.L.); kmjanggo@kookmin.ac.kr (J.J.); mijung@kookmin.ac.kr (M.L.) \\ * Correspondence: hyunjoo@kookmin.ac.kr
}

check for

updates

Citation: Song, K.; Son, H.; Park, S.; Lee, J.; Jang, J.; Lee, M.; Choi, H.-j.

Fabrication of Piezo-Resistance

Composites Containing

Thermoplastic Polyurethane/Hybrid Filler Using 3D Printing. Sensors 2021, 21, 6813. https://doi.org/10.3390/ s21206813

Academic Editors: Sung Hoon Kang and Zhiyong Xia

Received: 30 August 2021

Accepted: 6 October 2021

Published: 13 October 2021

Publisher's Note: MDPI stays neutral with regard to jurisdictional claims in published maps and institutional affiliations.

Copyright: (c) 2021 by the authors. Licensee MDPI, Basel, Switzerland. This article is an open access article distributed under the terms and conditions of the Creative Commons Attribution (CC BY) license (https:// creativecommons.org/licenses/by/ $4.0 /)$.

\begin{abstract}
In this study, 3D-printable flexible piezoresistive composites containing various amounts of cilia-like hybrid fillers were developed. In the hybrid fillers, micro-scale $\mathrm{Cu}$ particles with a OD structure may allow them to easily disperse into the flexible TPU matrix. Furthermore, nanoscale multi-walled carbon nanotubes (MWCNTs) with a high aspect ratio, present on the surface of the $\mathrm{Cu}$ particles, form an electrical network when the polymer matrix is strained, thus providing good piezoresistive performance as well as good flowability of the composite materials. With an optimal hybrid filler content (17.5 vol.\%), the 3D-printed piezoresistive composite exhibits a gauge factor of 6.04 , strain range of over $20 \%$, and durability of over 100 cycles. These results highlight the potential applications of piezoresistive pressure sensors for health monitoring, touch sensors, and electronic skin.
\end{abstract}

Keywords: conductive composites; 3D printing; hybrid filler; piezoresistive sensitivity; health monitoring; touch/flexible sensors

\section{Introduction}

Smart fashion has generated considerable interest in various fields such as commerce, medicine, military, and aerospace. Among wearable technologies, flexible piezoresistive materials have potential applications in strain/pressure sensors, touch sensors, electronic skin, and biomedical robotics [1].

One way to produce flexible piezoresistive materials is to utilize conductive polymer composites (CPCs), wherein a conductive network is formed inside the material during its deformation and, when a larger external force is applied, a change in the electrical response occurs. In the past few years, flexible CPCs with various fillers (i.e., metallic particles, conductive polymers, and carbon materials) have been reported. For example, several studies have reported an improvement in electrical conductivity by dispersing metallic pillars such as copper, gold, and silver in the form of nanoparticles, nanowires, and nanosheets [2]. However, the poor compatibility of the interface between the metallic fillers and polymer matrix can adversely affect the piezoresistive properties. Thus, owing to their compatibility with the matrix, the use of conductive polymers as fillers has been proposed by few researchers. Conductive polymer fillers such as poly(3,4-ethylenedioxythiophene): poly (styrene-sulfonate) (PEDOT: PSS), poly(styrene- $\beta$-isobutylene- $\beta$-styrene), and poly (3-hexylthiophene) are widely used to obtain stretchable and cost-efficient composites using various methods, such as chemical doping and dip coating [3-5]. Although PEDOT: PSS is well known as a suitable conductive polymer for a filler, its electrical conductivity (4380 S cm$~^{-1}$ ) is far lower than that of metallic or carbon-containing filler composites (5.96 $\times 10^{7} \mathrm{~S} \mathrm{~cm}^{-1}$ for copper and $10^{6}$ to $10^{7} \mathrm{~S} \mathrm{~cm}^{-1}$ for CNTs) [6]. Another candidate for CPCs is carbon nanofiller, which shows no delamination owing to its better compatibility compared to that of metal fillers and has a high electronic conductivity. Several studies have reported the use of carbon nanofillers with high aspect ratios (i.e., width-to-thickness ratios) 
to form conductive pathways in the insulating polymer matrix. These studies have demonstrated that polymer/CNTs or polymer/graphene composites have good piezoresistive performances [6-12]. However, carbon fillers generally tend to agglomerate owing to their affinity resulting from strong Van der Waals forces; this issue adversely affects the uniform dispersion of the carbon filler in the flexible matrix. Recently, many attempts have been made to fabricate polymer composites using a variety of processes, especially in the field of functional composite materials. For instance, using the chemical reaction of polyhedral oligomeric silsesquioxane (POSS) and halloysite nanotubes (HNTs), Wu et al. suggested that the inclusion of HNTs-POSS enhances the fire safety of TPU nanocomposites [10]. In addition, one recent study shows that polyvinylidene fluoride (PVDF) films containing graphene nanoplatelets (GNPs) are suitable for flexible strain sensor fabrication using the solution mixing method [11]. Recently, graphene-based piezoresistive materials with a 3D structure have been synthesized by various methods such as 3D printing, self-assembly, and electrospinning [12]. Although 3D structure has been known to greatly enhance sensor performance, the low-cost, large-scale synthesis of 3D-structured piezoresistive materials is still challenging.

Herein, to address the agglomeration problem of carbon nanomaterials in polymer matrix, we propose a new class of flexible piezoresistive materials containing MWCNTs/metal hybrid fillers. In our previous study, a cilia-like metal/CNTs hybrid powder was proposed that formed a 3D structure and exhibited good electrical conductivity in the polymer matrix [13]. In piezoresistive materials, hybrid fillers may form geometrically conductive networks when the polymer matrix is strained. This study describes the use of an additive manufacturing technique to enhance the design flexibility of the newly developed piezoresistive materials. Subsequently, the piezoresistive performance of the 3D-printed flexible composites containing monolithic and hybrid fillers is discussed with respect to the microstructures and electrical conductivities.

\section{Experimental}

\subsection{Materials and Methods}

$\mathrm{Cu} / \mathrm{MWCNT}$ hybrid powder was fabricated by mechanical milling of a mixture of $\mathrm{Cu}$ powder $(\mathrm{Cu}$, mean size $1 \mu \mathrm{m}( \pm 1.25)$, spherical shape; Kojundo Co. Ltd., Japan) and MWCNTs (diameter $\sim 20 \mathrm{~nm}$, length $\sim 10 \mu \mathrm{m}$, purity $>90 \%$; Applied Carbon Nano Technology Co. Ltd., Korea). The $\mathrm{Cu}$ and MWCNTs powders, as well as the ball-milled composite powder, are shown in Figure 1a. A planetary mill (Pulverisette 5; FRITSCH Inc., Germany) was used to uniformly disperse and partially insert MWCNTs on the surface of the micro-sized $\mathrm{Cu}$ particles. The weight ratio of the MWCNTs to $\mathrm{Cu}$ powder was 1:2. The milling process was conducted for $2 \mathrm{~h}$ at a milling speed of $200 \mathrm{rpm}$ and a powderto-ball weight ratio of 1:15. The composite powder was then mixed with thermoplastic polyurethane (TPU, round shape pellet; Shandong Huada Chemical New Material Co., Ltd., China) to produce pellets for 3D printing. TPU was dissolved in N,N-dimethylformamide (DMF) solution (Daejung Chem. Co. Ltd., Korea), and the $\mathrm{Cu} / \mathrm{MWCNT}$ hybrid powder was added to the TPU polymer solution and mixed using a magnetic stirrer for $4 \mathrm{~h}$ at room temperature to obtain a well-dispersed TPU/Cu/MWCNT solution. The solution was dried and then degassed in a solvent hood for $24 \mathrm{~h}$ to remove DMF. The volume fractions of the filler (i.e., $\mathrm{Cu} / \mathrm{MWCNTs}$ ) were 5, 15, 17.5, and 20\%. The TPU/Cu/MWCNT composite was cut to fabricate a filament for the 3D printer, as shown in Figure 1b. The chopped TPU/Cu/MWCNTs were then extruded using a mini-extruder (Filabot EX2, Filabot Co., USA) with a diameter of $1.75 \mathrm{~mm}$ where the melting temperature was in the range of $180-210{ }^{\circ} \mathrm{C}$. We prepared two types of 3D-printed composites to measure the electrical conductivity (Figure 1c, $10 \times 10 \times 1.1 \mathrm{~mm}$ ) and conducted an experiment for investigating the piezoresistive behavior (Figure 1d, $9.5 \times 9.5 \times 19 \mathrm{~mm}$ ). To prepare the 3D-printed TPU/Cu/MWCNT composites, the filaments were 3D printed (fused deposition modeling) using a commercial 3D printer (3DP; 3DP-310FB, HyVISION SYSTEM Inc., Seongnam, Korea). The processing parameters of the printed TPU/Cu/MWCNTs are listed in Table 1. 

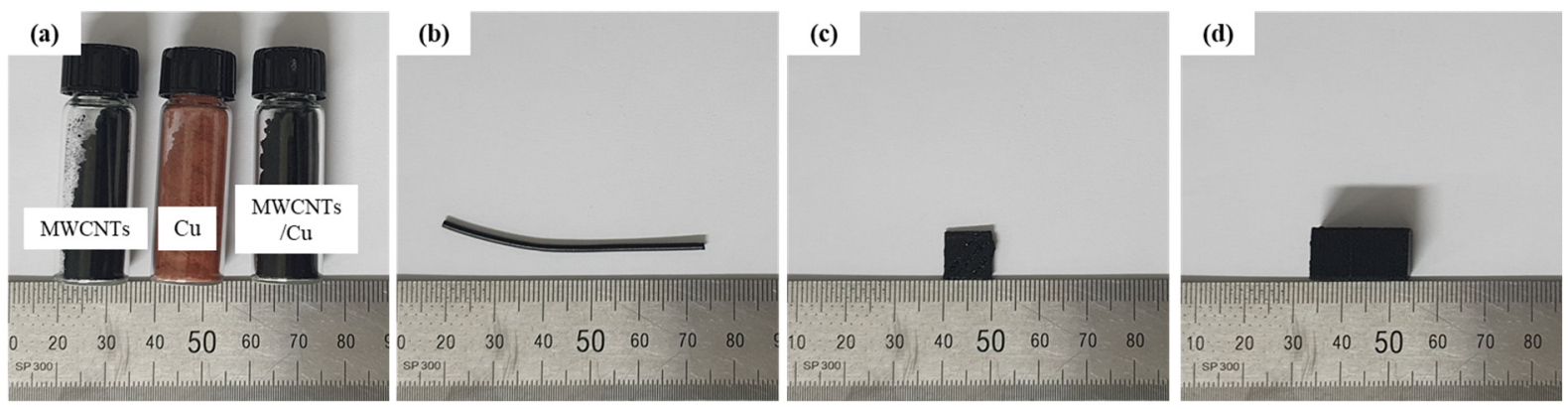

Figure 1. (a) Raw material images and the hybrid powder after mechanical milling; (b) extruded filaments of TPU/Cu/MWCNT composites before 3D printing; and the 3D-printed test specimen for (c) interrogation of the electrical conductivity and (d) compression test.

Table 1. 3D printing parameters to fabricate 3D-printed TPU/Cu/MWCNT composites.

\begin{tabular}{cc}
\hline Processing Parameter & Value \\
\hline Print nozzle diameter & $0.4 \mathrm{~mm}$ \\
Extruder temperature & $210^{\circ} \mathrm{C}$ \\
Bed temperature & $80^{\circ} \mathrm{C}$ \\
Chamber temperature & $35^{\circ} \mathrm{C}$ \\
Layer height & $0.2 \mathrm{~mm}$ \\
\hline
\end{tabular}

\subsection{Morphological, Electrical and Piezoresistive Characterization}

For microstructural morphology evaluations, a field emission scanning electron microscope (FE-SEM; JEM-7410F and JEM-7610f, JEOL Ltd., Japan) was used to observe the morphology of the $\mathrm{Cu}$ /MWCNT hybrid particle. An optical microscope (OM, Daemyung Co. Ltd., Korea) was used to observe the cross-sectional microstructure of the TPU/Cu/MWCNT hybrid filaments. Raman spectroscopy (Raman Spectrometer, LabRam ARAMIS, Horiba, Japan) was performed in the range of $1000-3000 \mathrm{~cm}^{-1}$. The 4-point-probe method was used to measure the sheet resistance of the 3D-printed TPU/Cu/MWCNT composites. The piezoresistive performance was tested using a universal tensile machine (UTM, RB 301 UNITECH-M, R\&B Inc., Daejeon, Korea) and a multimeter (DM3058E, RIGOL Technologies, Japan) to measure the change in the electrical resistance with respect to the applied tension. For this test, the 3D-printed compression specimen was loaded with a crosshead speed of $1 \mathrm{~mm} / \mathrm{min}$ using a 1-ton load cell. Copper tape $(10 \mathrm{~cm})$ was attached to the top and bottom sides of the 3D-printed compression specimen, which acted as electrodes. A $100 \times$ cyclic compress test was conducted under the same conditions as the compression test at displacements of 1 and $1.5 \mathrm{~mm}$ to illustrate the persistence of the piezoresistive performance.

\section{Results and Discussion}

\subsection{Surface and Cross-Sectional Morphology}

Figure 2 shows FE-SEM images of the MWCNTs, Cu powder, and ball-milled hybrid powder and its corresponding energy-dispersive spectrometer (EDS) map. The MWCNTs (Figure 2a) had an average diameter of $20 \mathrm{~nm}$ and were found to be curved and entangled. In contrast, the initial Cu powder was observed to be mostly spherical, but the sizes and shapes of the particles was irregular. The micro-sized $\mathrm{Cu}$ powder is difficult to plastically deform when mixed with entangled bundles of MWCNTs; therefore, the $\mathrm{Cu}$ powder was covered with MWCNTs, as shown in Figure 1c and its corresponding EDS map. As shown in Figure 2d-1,d-2, the hard and stiff MWCNTs partially embedded in the Cu powder and dispersed to some extent along with the plastic deformation of the $\mathrm{Cu}$ powder. 


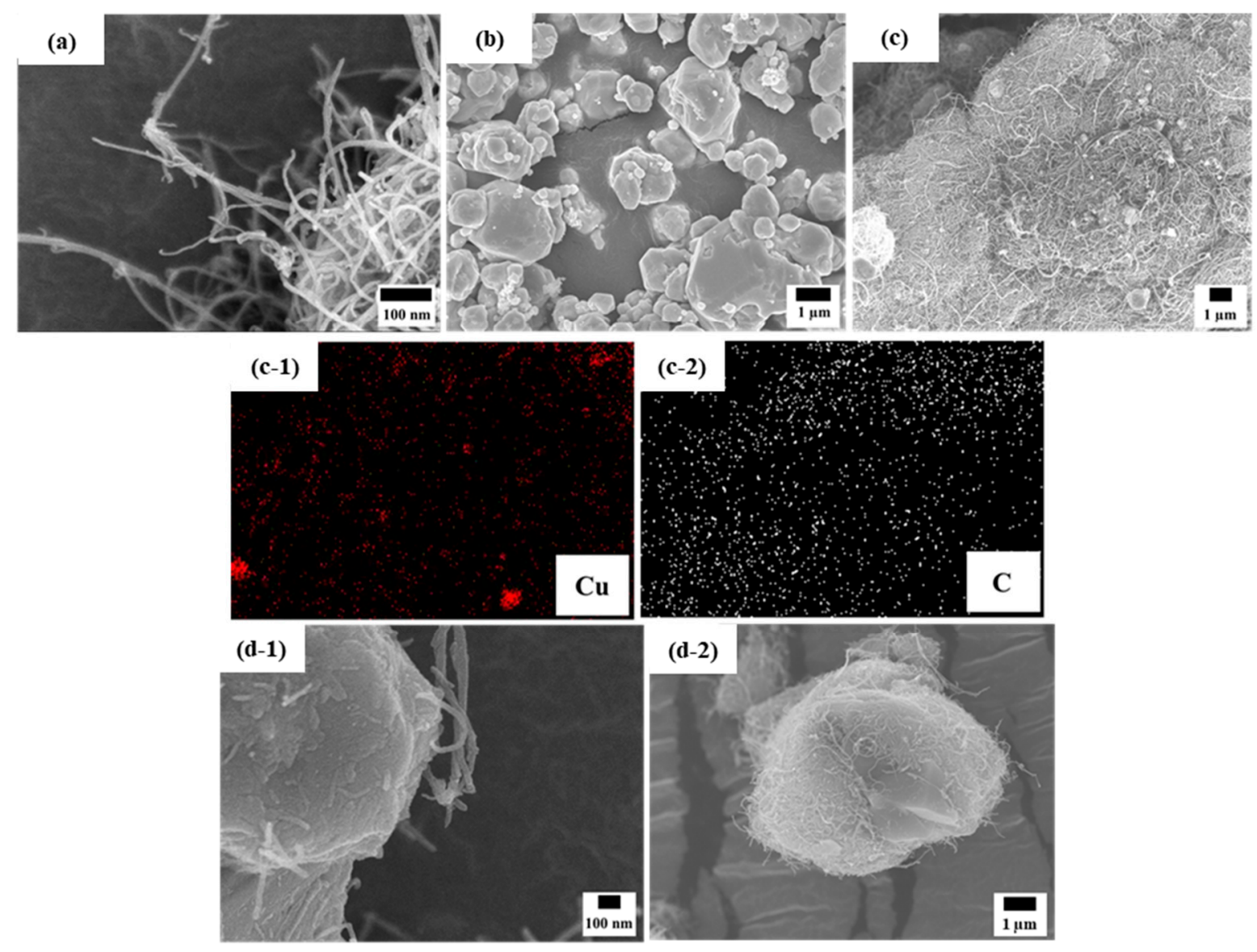

Figure 2. FE-SEM images of (a) initial MWCNTs, (b) initial Cu powder, (c) ball-milled hybrid powder and its corresponding EDS map (c-1,c-2), and (d-1,d-2) magnified images of the ball-milled hybrid powder wherein MWCNTs are partially embedded into $\mathrm{Cu}$ particles.

The ball-milled $\mathrm{Cu} / \mathrm{MWCNT}$ hybrid powder was then mixed with a flexible TPU; Figure 3a-c show optical images of the cross section of the hybrid filament. As shown, the bright particles (presumably the $\mathrm{Cu} / \mathrm{MWCNT}$ hybrid powder) were well dispersed in the flexible TPU matrix. Because the bright $\mathrm{Cu}$ particles in the OM images seem to be uniformly dispersed in the TPU matrix, it is assumed that the nano-sized MWCNTs, attached to the surface of the $\mathrm{Cu}$ particle (as shown in Figure 2), were also uniformly dispersed in this filament. The nano-sized MWCNTs are not seen in these OM images because of the low magnification. As the $\mathrm{Cu}$ /MWCNT hybrid powder content increased, the number of bright particles was also observed to increase, and the distance between each particle gradually decreased. The micro-scale $\mathrm{Cu}$ particles with a 0D structure may allow them to easily disperse into the flexible TPU matrix relative to the dispersion of nanoscale MWCNTs with a high aspect ratio. However, the MWCNTs, which cannot be easily dispersed solely in TPU, can be readily dispersed with the help of $\mathrm{Cu}$ powder because they cover or are embedded in the $\mathrm{Cu}$ powder, as shown in the schematic depiction of the microstructure of $\mathrm{Cu} / \mathrm{MWNCT} / \mathrm{TPU}$ pallets in Figure 3d. Thus, it can be expected that the 1D structure (i.e., a high aspect ratio) of the MWCNTs plays a pivotal role in the formation of conductive pathways surmounting the difficulty of dispersion of the nano-sized and entangled MWCNTs. 

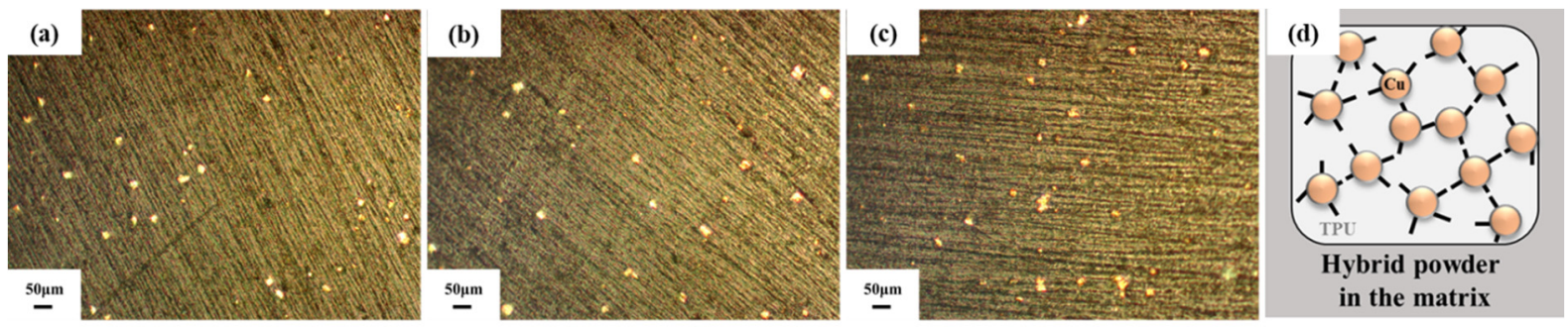

Figure 3. OM images of the TPU/Cu/CNTs filament cross section after extruding (a) 15, (b) 17.5, and (c) 20 vol.\%; (d) schematic of the hybrid particles in the matrix.

Raman spectra analysis is a useful method to examine the changes in the molecular structure of MWCNTs in the TPU matrix during mechanical milling and 3D printing processes. As shown in Figure 4, sharp and strong peaks appeared at $1580 \mathrm{~cm}^{-1}$, which is in the $G$ band, for all the samples, and its intensity was verified by the in-plane microstructure of the graphitic structure [14]. Other peaks at $\sim 1350 \mathrm{~cm}^{-1}$ and $\sim 1615 \mathrm{~cm}^{-1}$, which are in the $\mathrm{D}$ and $\mathrm{D}$ / bands, respectively, were also observed for all the samples. The ratio of the intensity of the D peak to that of the $G$ peak, which is used to infer the amount of structural defects in graphitic materials, increased from 0.60 to 0.87 after mechanical milling. The increase in the ratio of $\mathrm{I}_{\mathrm{D}} / \mathrm{I}_{\mathrm{G}}$ indicates that additional defects might have been created owing to mechanical milling. However, the $\mathrm{I}_{\mathrm{D}} / \mathrm{I}_{\mathrm{G}}$ ratio of $3 \mathrm{D}$-printed TPU $/ \mathrm{Cu} / \mathrm{MWCNT}$ composites decreased to 0.68 , which is comparable to that of the raw MWCNTs. During the extrusion and 3D printing, the specimens were exposed to high temperatures, which might have led to a recombination of the internal defects in the MWCNTs. Gong et al. have reported that the density of defects reduces during heat treatment [15]. Therefore, the molecular structure of MWCNTs is not significantly destroyed throughout the entire process.

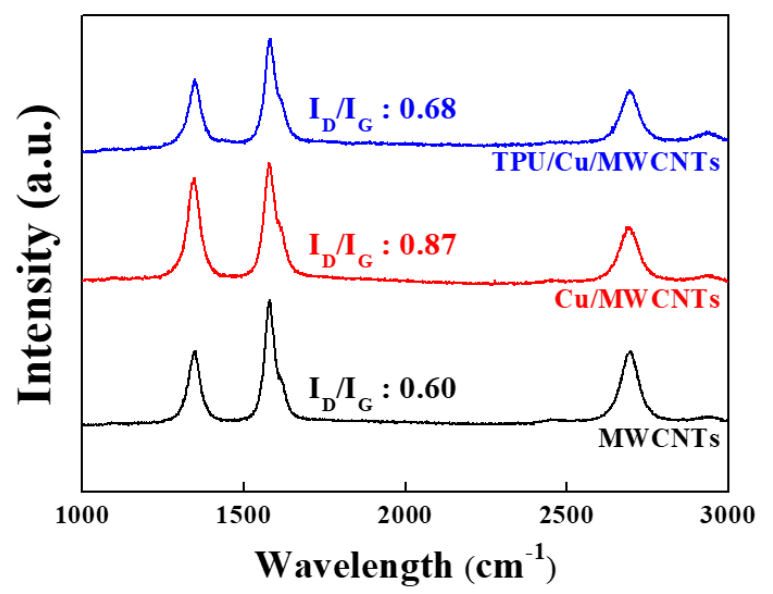

Figure 4. Polarized Raman spectra of TPU/Cu/MWCNTs, Cu/MWCNTs, and MWCNTs.

\subsection{Electrical and Piezoresistive Properties}

As shown in Figure 5a, the electrical properties of the 3D-printed TPU/Cu/MWCNT hybrid composites were compared with those of TPU composites dispersed with either $\mathrm{Cu}$ or MWCNTs, wherein, in all the cases, the amount of the filler was fixed at $5 \mathrm{vol} \%$. The sheet resistivity of the TPU/Cu, TPU/MWCNTs, and TPU/Cu/MWCNT hybrid composites was $1.0 \times 10^{5} \Omega \mathrm{m}, 8.7 \times 10^{4} \Omega \mathrm{m}$, and $7.9 \times 10^{4} \Omega \mathrm{m}$, respectively. MWCNTs were found to be more effective than micro-sized $\mathrm{Cu}$ particles as conducting agents because the presence of nano-sized 1D MWCNTs with a high aspect ratio enables the formation of electron pathways, compared to micro-sized and spherical $\mathrm{Cu}$. However, owing to the presence of Van der Waals forces, entangled MWCNTs cannot be easily dispersed in the TPU matrix. Because MWCNTs can be uniformly dispersed with the help of micro-sized $\mathrm{Cu}$ 
particles, the composites containing $\mathrm{Cu}$ /MWCNT hybrid fillers exhibited better electrical conductivity (lower sheet resistivity) than those containing solely dispersed MWCNTs. These $\mathrm{Cu}$ /MWCNT hybrid fillers may effectively form a conductive network. When the MWCNTs are uniformly dispersed, electrons may readily move from one MWCNT tip to another because of the tunneling effect. Figure $5 \mathrm{~b}$ shows the resistivity of the 3D-printed TPU/Cu/MWCNTs with different volume fractions of $\mathrm{Cu} / \mathrm{MWCNT}$ fillers, which was then used to determine the optimized volume fraction of the fillers required for $3 \mathrm{D}$ printing. The electrical resistivity of the specimens exponentially decreased with an increase in the volume fraction of the fillers, exhibiting a typical percolation threshold [16]. Referring to the OM image in Figure 3, it was deduced that when the distance between the hybrid particles in the TPU matrix decreased, the electrical conductivity was enhanced, owing to an increase in the conductive pathways such as electron tunneling. The electrical resistivity of the composites developed in the present study was also compared with that of a commercial piezoresistive pressure sensing fabric (EeonTex ${ }^{\mathrm{TM}}$ NW170-SLPA-2k, SparkFun Electronics, Niwot, CO, USA). When the filler content was greater than $15 \mathrm{vol} \%$, the resistivity was close to, or lower than, that of the commercial piezoresistive fabric. It is worth noticing that, along with the advantage of it being suitable for 3D printing, the new material developed in the present study shows superior electrical conductivity compared to that of commercial piezoresistive materials. This is important because the rheological properties of nanocomposite materials are known to deteriorate during fused deposition modeling (FDM) type $3 \mathrm{D}$ printing.
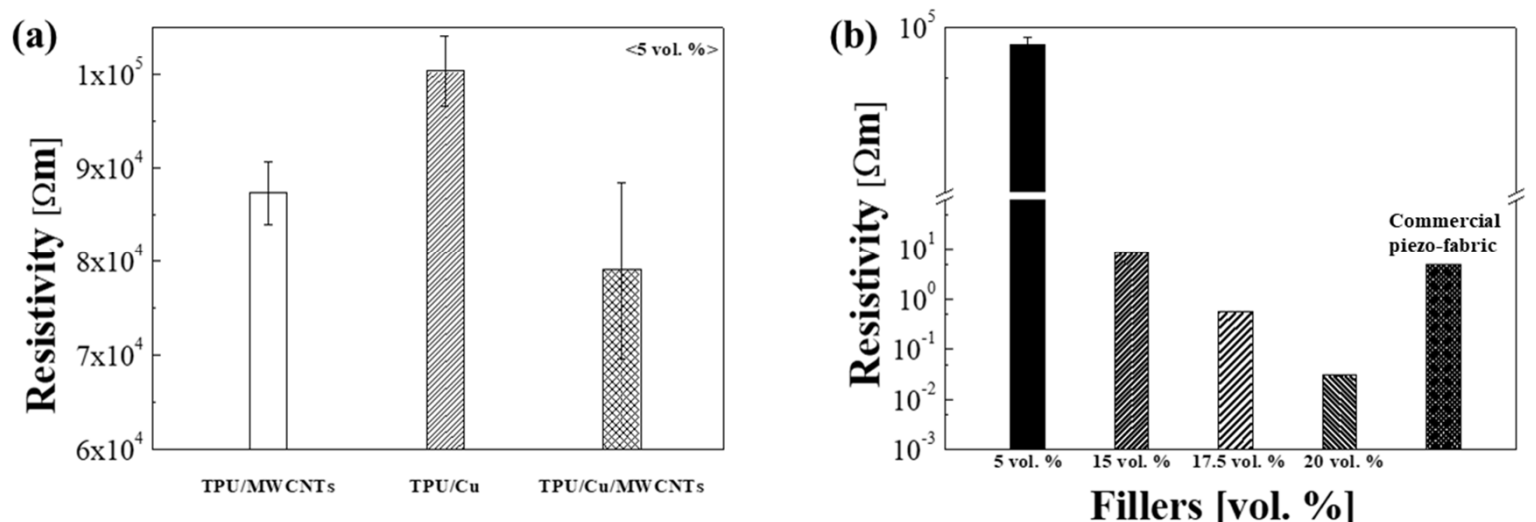

Figure 5. (a) Resistivity of the different fillers containing TPU at the same volume fraction; (b) resistivity of the 3D-printed $\mathrm{TPU} / \mathrm{Cu} / \mathrm{MWCNTs}$ compared with commercial piezoresistive fabric at different volume fractions.

The piezoresistive behavior of the 3D-printed hybrid composites was investigated via in situ compression tests, wherein the ratio of the initial resistivity $\left(\mathrm{R}_{0}\right)$ and resistivity change ( $\Delta R ; \Delta R=R-R_{0}$, where $R$ is the resistivity at a given strain) was monitored in real time till the composites were strained up to $20 \%(\varepsilon \leq 20 \%$, where $\varepsilon$ is the true strain during compression) or fractured (fracture strain was $8.7 \%$ ), and the results are shown in Figure $6 \mathrm{a}-\mathrm{c}$, respectively. The sensitivity of a piezoresistive material can be quantified through the gauge factor, $G$, using the following formula:

$$
\mathrm{G}=\left(\Delta \mathrm{R} / \mathrm{R}_{0}\right) / \varepsilon \ldots
$$



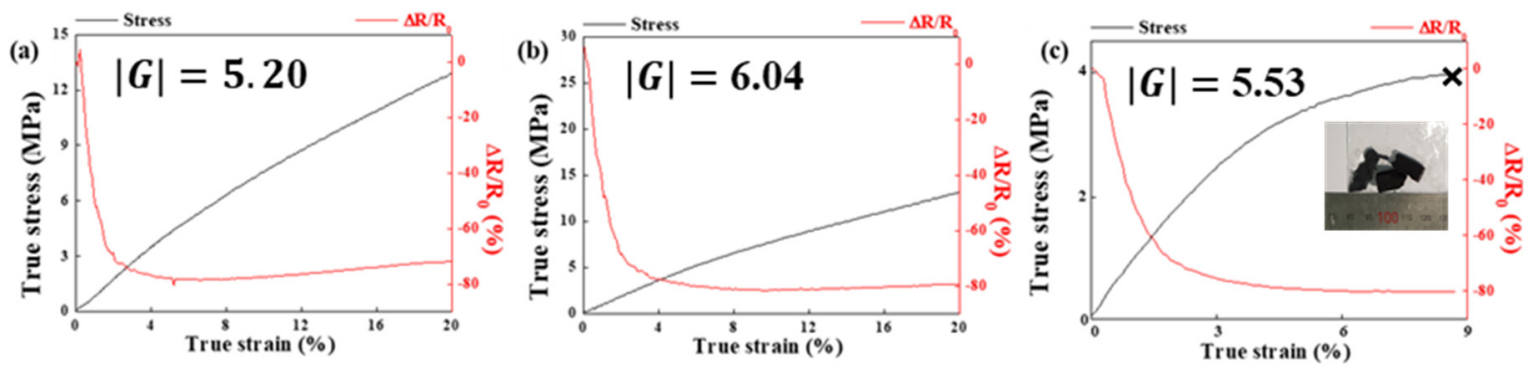

Figure 6. Normalized change of piezoresistive sensitivity and compressive stress versus strain for TPU/Cu/MWCNT composites at (a) 15 vol.\%, (b) 17.5 vol.\%, and (c) 20 vol. \%.

$\mathrm{G}$ can be defined as the slope of the $\Delta R / R_{0}$ curve during compression. For convenience of comparison, the absolute values of $G$ were used in the present study. The applied compressive true stress and the $\Delta R / R_{0}$ values were plotted against the compressive true strain. The gauge factor in the strain range of $0-8.7 \%$ was calculated. The gauge factors of the TPU/Cu/MWCNT hybrid composites with filler contents of $15,17.5$, and 20 vol.\% were measured as $5.20,6.04$, and 5.53 , respectively. The $\Delta R / R_{0}$ curves show a similar tendency of exponentially decreasing piezoresistive sensitivity for all the specimens in the strain range up to $5 \%$. During the initial stage of compression (strain $<5 \%$ ), conducting pathways can be easily formed with deformation as conducting fillers get closer and assemble. Once the percolation threshold was reached, the piezoresistive sensitivity value saturated with further straining. In the present study, MWCNTs may help the Cu hybrid particles form continuous conductive networks under compressive strain. In terms of the value of the gauge factor, an applied deformation to the 17.5 vol.\% sample was observed to induce strong and reversible variations in the conductive network configuration, which lead to a larger variation in the electrical conductivity. However, for composites with higher filler content ( $>20$ vol.\%), the piezoresistive sensitivity was observed to deteriorate, possibly because of the low sample quality (e.g., internal pores or defects) stemming from the low fluidity of the samples during printing. Furthermore, the disruption or modification of even a single conductive path may also deteriorate the piezoresistive sensitivity.

The reproducibility of piezoresistive sensitivity to strain during 100 cycles was also tested for the 17.5 vol.\% hybrid filler (Figure $7 \mathrm{a}, \mathrm{b}$ ). This cycling test was conducted in the compression strain range of $5-7 \%(1-1.5 \mathrm{~mm}$ displacement). For the first 10 cycles, the $\Delta \mathrm{R} / \mathrm{R}_{0}$ value reached approximately -60 to $-80 \%$ in a linear fashion and did not recover to its initial value (Figure 7a); this may have occurred because of the rearrangement of the hybrid fillers in the flexible TPU matrix. When the $\Delta R / R_{0}$ value reached -60 to $-80 \%$, the change in amplitude of $\Delta R / R_{0}$ was insignificant, and the hybrid fillers formed a conductive network after rearrangement. The true stress decreased significantly for the first 10 cycles and then stabilized (Figure 7b), possibly because of the viscoelastic properties of the TPU matrix $[17,18]$. In the first 5 loading cycles of the composites containing hybrid fillers, $\Delta \mathrm{R} / \mathrm{R} 0$ initially decreased and subsequently increased (Figure $7 \mathrm{c}$ ). In contrast, $\Delta \mathrm{R} / \mathrm{R} 0$ was observed to stabilize in the last 5 cycles (Figure $7 \mathrm{~d}$ ). During the initial loading stage, the reinforcement may rearrange to form a more effective electrical network; hence, the $\Delta R / R 0$ values may fluctuate. As reported elsewhere, the $\Delta R / R 0$ values can be stabilized once the filler network is stable [19]. 

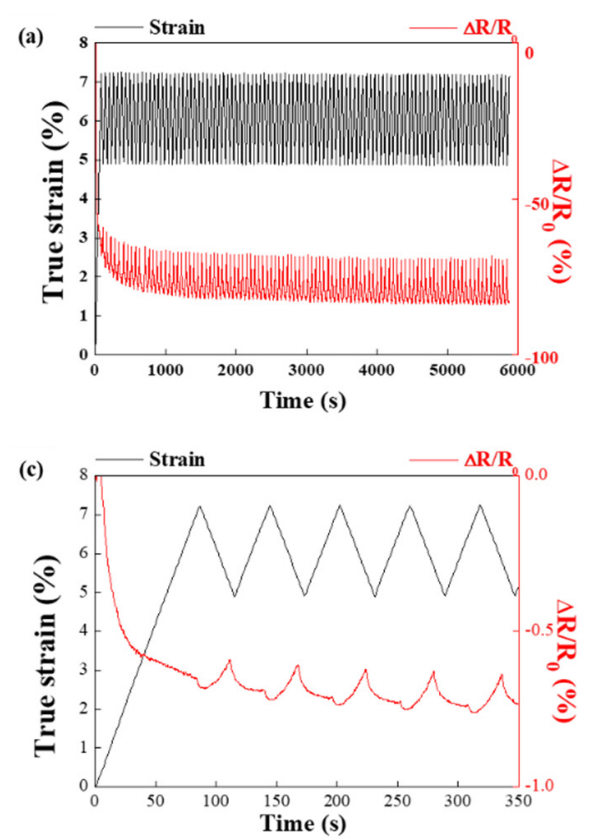
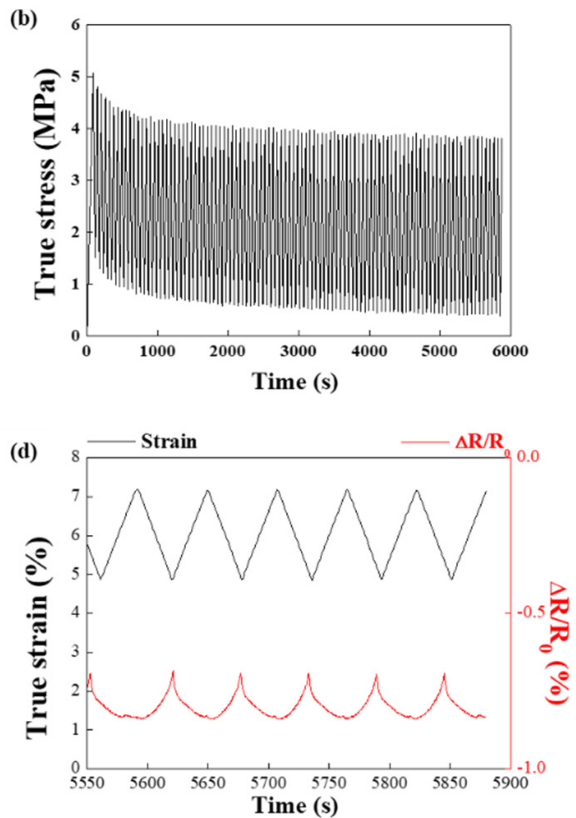

Figure 7. $(\mathbf{a}, \mathbf{b})$ Compression testing of 3D-printed TPU/Cu/MWCNT composites up to 100 cycles (c) first and (d) last 5 cycles.

\section{Conclusions}

When the filler content was greater than 15 vol. $\%$, the resistivity was close to that of a commercial piezoresistive fabric. It is worth noticing that the new material developed in the present study shows superior electrical conductivity compared to that of commercial piezoresistive materials, along with it being suitable for 3D printing. This is important because the rheological properties of nano-composite materials are known to deteriorate during fused deposition modeling (FDM) type 3D printing.

In this study, flexible TPU-based 3D printable composites containing hybrid fillers, which consist of $\mathrm{Cu}$ covered or partially embedded with MWCNTs, were prepared using the solution mixing method. Composites containing $5 \mathrm{wt}$ \% hybrid fillers exhibited higher electrical conductivity than those containing the same content of monolithic fillers, which demonstrates that hybrid fillers are beneficial for forming electrical networks. Furthermore, the hybrid fillers allow them to easily disperse into the flexible polymer due to its structural feature. Thus, owing to their microstructural characteristics, the 3D printable TPU/Cu/MWCNT composites showed better electrical conductivity than TPU composites dispersed with only $\mathrm{Cu}$ or MWCNTs. As the hybrid filler content increased, a higher gauge factor was obtained. However, the composites with the highest filler content (20 vol.\%) showed rather deteriorated and lower gauge factors. In the case of the optimized composites (17.5 vol.\%), the 3D-printed TPU/Cu/MWCNT composites showed a gauge factor of 6.04 and a strain range of over $20 \%$. Furthermore, it is worth noticing that 3D printable composites containing the hybrid filler showed superior electrical conductivity than a commercial piezoresistive fabric. Finally, when using a proper amount of filler in the matrix during the 3D printing process, the TPU/Cu/MWCNT composites showed excellent electrical conductivity while maintaining flexibility and are thus a good candidate for electrical resistive materials in smart fashion.

Author Contributions: Conceptualization, H.-j.C. and K.S.; methodology, H.-j.C. and K.S.; software, K.S.; validation, H.-j.C., J.J., M.L. and K.S.; formal analysis, K.S.; investigation, K.S., H.S., S.P. and J.L.; resources, K.S.; data curation, K.S., H.S., S.P. and J.L.; writing-original draft preparation, K.S.; writing-review and editing, H.-j.C., J.J., M.L. and K.S.; visualization, K.S.; supervision, H.-j.C.; project administration, H.-j.C.; funding acquisition, H.-j.C. All authors have read and agreed to the published version of the manuscript. 
Funding: This work was supported by the National Research Foundation of Korea (NRF) Grant funded by the Korean Government (MSIP), Grant No. 2015R1A7037615.

Institutional Review Board Statement: Not applicable.

Informed Consent Statement: Not applicable.

Conflicts of Interest: The authors declare no conflict of interest. The funders had no role in the design of the study; in the collection, analyses, or interpretation of data; in the writing of the manuscript, or in the decision to publish the results.

\section{References}

1. Castano, L.M.; Flatau, A.B. Smart fabric sensors and e-textile technologies: A review. Smart Mater. Struct. 2014, $23,053001$. [CrossRef]

2. Fiorillo, A.S.; Critello, C.D.; Pullano, S.A. Theory, technology and applications of piezoresistive sensors: A review. Sens. Act. A 2018, 281, 156-175. [CrossRef]

3. Zhou, Z.; Zhang, X.; Wu, X.; Lu, C. Self-stabilized polyaniline@graphene aqueous colloids for the construction of assembled conductive network in rubber matrix and its chemical sensing application. Compos. Sci. Technol. 2018, 125, 1-8. [CrossRef]

4. Tian, M.; Wang, Y.; Qu, L.; Zhu, S.; Han, G.; Zhang, X.; Zhou, Q.; Du, M.; Chi, S. Electromechanical deformation sensors based on polyurethane/polyaniline electrospinning nanofibrous mats. Synth. Metals 2016, 219, 11-19. [CrossRef]

5. Wang, J.P.; Xue, P.; Tao, X.M. Strain sensing behavior of electrically conductive fibers under large deformation. Mat. Sci. Eng. A 2011, 528, 2863-2869. [CrossRef]

6. Shi, H.; Liu, C.; Jiang, Q.; Xu, J. Effective approaches to improve the electrical conductivity of PEDOT: PSS: A review. Adv. Electron. Mater. 2015, 1, 1500017. [CrossRef]

7. Xiang, D.; Wang, L.; Tang, Y.; Zhao, C.; Harkin-Jones, E.; Li, Y. Effect of phase transitions on the electrical properties of polymer/carbon nanotube and polymer/graphene nanoplatelet composites with different conductive network structures. Polym. Int. 2018, 67, 227-235. [CrossRef]

8. Araby, S.; Meng, Q.; Zhang, L.; Kang, H.; Majewski, P.; Tang, Y.; Ma, J. Electrically and thermally conductive elastomer/graphene nanocomposites by solution mixing. Polymer 2014, 55, 201-210. [CrossRef]

9. Rinaldi, A.; Tamburrano, A.; Fortunato, M.; Sarto, M.S. A flexible and highly sensitive pressure sensor based on a PDMS foam coated with graphene nanoplatelets. Sensors 2016, 16, 2148. [CrossRef] [PubMed]

10. Wu, W.; Zhao, W.; Gong, X.; Sun, Q.; Cao, X.; Su, Y.; Yu, B.; Li, R.K.; Vellaisamy, R.A. Surface decoration of halloysite nanotubes with POSS for fire-safe thermoplastic polyurethane nanocomposites. J. Mat. Sci. Technol. 2021, 101, 107-117. [CrossRef]

11. Cheraghi Bidsorkhi, H.; D'Aloia, A.G.; Tamburrano, A.; De Bellis, G.; Sarto, M.S. Waterproof Graphene-PVDF Wearable Strain Sensors for Movement Detection in Smart Gloves. Sensors 2021, 21, 5277. [CrossRef] [PubMed]

12. Cao, M.; Su, J.; Fan, S.; Qiu, H.; Su, D.; Li, L. Wearable piezoresistive pressure sensors based on 3D graphene. Chem. Eng. J. 2021, 406, 126777. [CrossRef]

13. Cho, M.; Choi, H.; Aslam, M.; Shin, J.; Bae, D. Electrically conductive epoxy composites containing cilia-like powders fabricated by mechanical milling. Met. Mater. Int. 2010, 16, 67-70. [CrossRef]

14. Darsono, N.; Yoon, D.H.; Kim, J. Milling and dispersion of multi-walled carbon nanotubes in texanol. Appl. Surf. Sci. 2008, 254, 3412-3419. [CrossRef]

15. Gong, Q.M.; Li, Z.; Wang, Y.; Wu, B.; Zhang, Z.; Liang, J. The effect of high-temperature annealing on the structure and electrical properties of well-aligned carbon nanotubes. Mater. Res. Bull. 2007, 42, 474-481. [CrossRef]

16. Wang, Y.; Shan, J.W.; Weng, G.J. Percolation threshold and electrical conductivity of graphene-based nanocomposites with filler agglomeration and interfacial tunneling. J. Appl. Phys. 2015, 118, 065101. [CrossRef]

17. Zhai, T.; Li, D.; Fei, G.; Xia, H. Piezoresistive and compression resistance relaxation behavior of water blown carbon nanotube/polyurethane composite foam. Compos. A 2015, 72, 108-114. [CrossRef]

18. Fei, Y.; Chen, F.; Fang, W.; Xu, L.; Ruan, S.; Liu, X.; Zhong, M.; Kuang, T. High-strength, flexible and cycling-stable piezo-resistive polymeric foams derived from thermoplastic polyurethane and multi-wall carbon nanotubes. Compos. B 2020, $199,108279$. [CrossRef]

19. Zha, J.-W.; Zhang, B.; Li, R.K.Y.; Dang, Z.-M. High-performance strain sensors based on functionalized graphene nanoplates for damage monitoring. Compos. Sci. Technol. 2016, 123, 32. [CrossRef] 